\title{
Relic density of dark matter in the inert doublet model beyond leading order: The heavy mass case
}

\author{
Shankha Banerjee $\odot,{ }^{1, *}$ Fawzi Boudjema $\odot,{ }^{2, \dagger}$ Nabarun Chakrabarty, ${ }^{3, \$}$ Guillaume Chalons, ${ }^{4, \S}$ and Hao Sun ${ }^{5, \|}$ \\ ${ }^{1}$ Institute for Particle Physics Phenomenology, Department of Physics, Durham University, \\ Durham DH1 3LE, United Kingdom \\ ${ }^{2}$ LAPTh, Université Savoie Mont Blanc, CNRS, BP 110, F-74941 Annecy-le-Vieux, France \\ ${ }^{3}$ Physics Division, National Center for Theoretical Sciences, Hsinchu, Taiwan 30013, Republic of China \\ ${ }^{4}$ Laboratoire de Physique Subatomique et de Cosmologie, \\ Université Grenoble-Alpes, CNRS/IN2P3, 53 Avenue des Martyrs, F-38026 Grenoble, France \\ ${ }^{5}$ Institute of Theoretical Physics, School of Physics, Dalian University of Technology, \\ Dalian 116024, People's Republic of China
}

(Received 10 July 2019; published 25 November 2019)

\begin{abstract}
A full renormalization of the inert doublet model is presented and exploited for a precise calculation of the relic density of dark matter (DM) at one-loop. In this first paper, we study the case of a DM candidate with $m_{\mathrm{DM}} \sim 500 \mathrm{GeV}$. In this regime, the coannihilation channels are important. We therefore compute, for a wide range of relative velocities, the full next-to-leading order electroweak corrections to seven annihilation/coannihilation processes that contribute $\sim 70 \%$ to the relic density of DM. These corrected cross sections are interfaced with microMEGAs to obtain the one-loop correction to the freeze-out relic density. Due to the accurate measurement of this observable, the one-loop corrections are relevant. We discuss the one-loop renormalization scheme dependence and point out the influence, at one-loop, of a parameter that solely describes the scattering in the dark sector. A tree-level computation of the relic density is not sensitive to this parameter.
\end{abstract}

DOI: 10.1103/PhysRevD.100.095024

\section{INTRODUCTION}

The inert doublet model (IDM) consists of adding a scalar doublet, $\Phi_{2}$, to the Standard Model (SM) of particle physics [1]. Endowing this most simple extension of the SM with an unbroken $\mathbb{Z}_{2}$ symmetry where $\Phi_{2}$ is odd while other fields of the SM are even, guarantees the stability of the lightest inert particle, thus providing a possible dark matter (DM) candidate [2]. The new scalars of this additional doublet couple to the Higgs and the gauge bosons but not to the fermions in the SM. This model provides a nice link between the Higgs sector with the source of electroweak symmetry breaking and DM [3]. The model has received a lot of attention, primarily for DM studies but also for collider observables; see [4-10] for reviews and

\footnotetext{
*shankha.banerjee@durham.ac.uk

boudjema@lapth.cnrs.fr

*nchakrabarty@cts.nthu.edu.tw

\$chalons@1psc.in2p3.fr

haosun@dlut.edu.cn
}

Published by the American Physical Society under the terms of the Creative Commons Attribution 4.0 International license. Further distribution of this work must maintain attribution to the author(s) and the published article's title, journal citation, and DOI. Funded by SCOAP ${ }^{3}$. updates. The majority of these analyses were performed at leading order. A few exceptions where one-loop effects are considered include (i) the computation of the trilinear self-coupling of the SM-like Higgs boson [11-14], (ii) oneloop corrections to the Higgs effective potential $[3,15]$, the running of the Higgs/scalar masses and the running of the scalar parameters [6] (see also [16]), (iii) one-loop induced cross sections relevant for direct detection [17] and (iv) induced one-loop effects for photon production: Higgs decay to a photon pair [18] and DM annihilation to photons $[19,20]$. Yet, the relic density of DM as extracted by Planck [21] is a precision measurement at the percent level that calls for an equally precise theoretical prediction. In particular, the perturbative DM annihilation cross sections that drive the amount of relic density must be evaluated beyond tree level. This has not been performed in the IDM case despite the fact that the relic density sets the most stringent constraint on the IDM. This is somehow understandable since this task requires a coherent full renormalization of the model, the evaluation of many processes at one-loop order and the inclusion of these processes for the evaluation of the relic density. It is the purpose of this paper to present such a program and to present the first results for one-loop-corrected processes and how they affect the value of the freeze-out relic density in the IDM. 
The IDM model is of course subject to various experimental constraints that leave two viable scenarios: one where the DM mass is about $M_{W}$ and the other where the mass is above $500 \mathrm{GeV}$. We will, in this first paper, be interested in a scenario with $M_{\mathrm{DM}} \sim 500 \mathrm{GeV}$. Nonperturbative effects, such as the importance of the electroweak Sommerfeld effects [5,22-25], occur for very high masses above the $\mathrm{TeV}$ and will not be treated here.

The plan of the paper is as follows. In the next Sec. II we will first outline the model and underline its parameters. The renormalization procedure is exposed in Sec. III. Section IV takes into account the various constraints on the model and motivates us in setting up our benchmark scenario. Section V presents our findings for the full nextto-leading order corrections that, at tree level, contribute more than $5 \%$ of the relic density contribution. We will find that because of coannihilation we will need to consider seven processes. Section VI will translate these improved predictions into a corrected value of the relic density by interfacing our cross sections with micrOMEGAs. Finally we conclude our findings in Sec. VIII. Appendixes are also provided.

\section{THE INERT DOUBLET MODEL AT THE CLASSICAL LEVEL}

To the Higgs doublet $\Phi_{1}$ of the SM, a doublet $\Phi_{2}$ is added. An unbroken $\mathbb{Z}_{2}$ symmetry is imposed under which $\Phi_{2}$ is odd while all other fields (of the SM) are even. The immediate consequence is that $\Phi_{2}$ cannot couple to fermions to any order (in perturbation theory) and guarantees the stability of the lightest inert particle, thus providing a possible dark matter candidate. The Lagrangian of the IDM can be written as

$$
\mathcal{L}_{\mathrm{IDM}}=\mathcal{L}_{\mathrm{SM}}+\left(D^{\mu} \Phi_{2}\right)^{\dagger} D_{\mu} \Phi_{2}+\mathcal{V}_{\mathrm{IDM}}\left(\Phi_{1}, \Phi_{2}\right),
$$

where $\mathcal{L}_{\mathrm{SM}}$ is the SM Lagrangian whereas the scalar potential is given by

$$
\begin{aligned}
\mathcal{V}_{\mathrm{IDM}}\left(\Phi_{1}, \Phi_{2}\right)= & \mu_{1}^{2}\left|\Phi_{1}\right|^{2}+\mu_{2}^{2}\left|\Phi_{2}\right|^{2}+\lambda_{1}\left|\Phi_{1}\right|^{4}+\lambda_{2}\left|\Phi_{2}\right|^{4} \\
& +\lambda_{3}\left|\Phi_{1}\right|^{2}\left|\Phi_{2}\right|^{2}+\lambda_{4}\left(\Phi_{2}^{\dagger} \Phi_{1}\right)\left(\Phi_{1}^{\dagger} \Phi_{2}\right) \\
& +\left(\frac{\lambda_{5}}{2}\left(\Phi_{1}^{\dagger} \Phi_{2}\right)^{2}+\text { H.c. }\right)
\end{aligned}
$$

In this equation, $\mu_{i}$ and $\lambda_{i}$ are real. Since the unbroken $\mathbb{Z}_{2}$ symmetry prevents the presence of tadpole terms for $\Phi_{2}$ (and therefore no vacuum expectation value from $\Phi_{2}$ ) and mixing with $\Phi_{1}$, we can directly parametrize the doublets in terms of the physical scalars,
$\Phi_{1}=\left(\begin{array}{c}G^{+} \\ \frac{1}{\sqrt{2}}(v+h+i G)\end{array}\right)$ and $\Phi_{2}=\left(\begin{array}{c}H^{+} \\ \frac{1}{\sqrt{2}}(H+i A)\end{array}\right)$,

where $v$ is the SM vacuum expectation value with $v \simeq$ $246 \mathrm{GeV}$ defined from the measurement of the $W\left(M_{W}\right)$ and $Z\left(M_{Z}\right)$ masses. We have

$$
s_{W}^{2} \equiv \sin ^{2} \theta_{W}=1-\frac{M_{W}^{2}}{M_{Z}^{2}}, \quad M_{W}=\frac{1}{2} \frac{e}{s_{W}} v,
$$

$e$ is the electromagnetic coupling [the $S U(2)$ gauge coupling $g$ is then $\left.g=e / s_{W}\right], h$ is the SM $125 \mathrm{GeV}$ Higgs boson, $G, G^{ \pm}$are the Goldstone bosons, $H, A$ are the new neutral physical scalars ${ }^{1}$ and $H^{ \pm}$is the charged physical scalar. $H$ and $A$ are the possible DM candidates. These scalars have gauge couplings to the SM gauge bosons controlled by the $\mathrm{SM}$ gauge coupling. For example, for the trilinear couplings we have

$$
\begin{gathered}
\left(H^{+} H^{-} \gamma, H^{+} H^{-} Z, H H^{ \pm} W^{\mp}, i A H^{ \pm} W^{\mp}, i A H Z\right) \\
\quad=i \frac{g}{2}\left(2 s_{W}, c_{2 W} / c_{W}, \mp 1,-1,-1 / c_{W}\right) .
\end{gathered}
$$

We must note that quartic couplings of the type $H H W^{+} W^{-}$are also present. Annihilation of DM to vector bosons proceeds, in part, through these gauge interactions and in part through the scalar potential coupling to which we now turn our attention for more details.

\section{A. Minimization of the potential}

Minimization of the potential amounts to vanishing tadpoles for $\Phi_{1}$ leading to the constraint

$$
\frac{T}{v}=\mu_{1}^{2}+\lambda_{1} v^{2} \equiv 0
$$

There is no corresponding tadpole term for $\Phi_{2}$ because of the unbroken $\mathbb{Z}_{2}$ symmetry. The no-tadpole condition will be maintained at all orders.

\section{B. Mass spectrum and scalar self-interactions}

By collecting the bilinear terms in the physical scalar fields of $\mathcal{V}_{\text {IDM }}$, we get the mass spectrum of the scalar sector of the IDM

$$
M_{h}^{2}=\frac{T}{v}+2 \lambda_{1} v^{2},
$$

\footnotetext{
${ }^{1}$ Since these additional scalars do not couple to the fermions (of the SM), we cannot assign them definite $C P$ numbers. By an abuse of language, we will call $A$ the pseudoscalar.
} 


$$
\begin{gathered}
M_{H^{ \pm}}^{2}=\mu_{2}^{2}+\lambda_{3} \frac{v^{2}}{2} \\
M_{H}^{2}=\mu_{2}^{2}+\lambda_{L} \frac{v^{2}}{2}=M_{H^{ \pm}}^{2}+\left(\lambda_{4}+\lambda_{5}\right) \frac{v^{2}}{2} \\
M_{A}^{2}=\mu_{2}^{2}+\lambda_{A} \frac{v^{2}}{2}=M_{H^{ \pm}}^{2}+\left(\lambda_{4}-\lambda_{5}\right) \frac{v^{2}}{2}=M_{H}^{2}-\lambda_{5} v^{2}
\end{gathered}
$$

where

$$
\lambda_{L / A}=\lambda_{3}+\lambda_{4} \pm \lambda_{5}
$$

In the following, we will consider $H$ as the possible DM candidate. The underlying reason is that both $H$ and $A$ can be treated on equal footing. Choosing $A$ as the DM candidate would simply correspond to a flip in the sign $\lambda_{5} \rightarrow-\lambda_{5}$ without changing the phenomenology. The reason is the following (we borrow arguments from [26]): Taking $H$ as the DM candidate and thus $M_{H}<M_{A}, M_{H^{ \pm}}$, from Eqs. (2.9) and (2.10) we obtain

$$
\lambda_{4}+\lambda_{5}<0 \text { and } \lambda_{5}<0
$$

The converse situation, with $M_{A}<M_{H}, M_{H^{ \pm}}$, corresponds to

$$
\lambda_{4}-\lambda_{5}<0 \text { and } \lambda_{5}>0
$$

For $\lambda_{5}=0, H$ and $A$ are mass degenerate. All portal triple and quartic couplings of the SM-like Higgs $h$ to $H / A$ are proportional to $\lambda_{L / A}$. Indeed, we can write, at tree level, the $h H H$ and $h A A$ coupling as

$$
\lambda_{h H H}=\lambda_{L} v, \quad \lambda_{h A A}=\lambda_{A} v
$$

Thus considering one or the other scalars as the DM candidate amounts to switching

$$
\lambda_{5} \leftrightarrow-\lambda_{5}, \quad \lambda_{L} \leftrightarrow \lambda_{A}
$$

In the same vein, we can write

$$
\lambda_{h H^{+} H^{-}}=\lambda_{3} v
$$

The quartic couplings between the SM Higgs and the new scalar are set by $\lambda_{3, L, A}$,

$$
\lambda_{h h H H, h h A A, h h H^{+} H^{-}}=\lambda_{L}, \lambda_{A}, \lambda_{3} .
$$

On the other hand, $\lambda_{2}$ controls all the quartic couplings solely within the dark sector $\left(\mathrm{HHHH}, \mathrm{HHAA}, \mathrm{HHH}^{+} \mathrm{H}^{-}\right.$, $A A A A, A A H^{+} H^{-}$and $\left.H^{+} H^{-} H^{+} H^{-}\right)$.

\section{Counting parameters}

In order to survey the IDM parameter space it is important to count the number of independent parameters in the scalar sector. Setting aside the tadpole condition and the $125 \mathrm{GeV}$ (SM) Higgs mass, the IDM requires five extra parameters,

$$
\left(\mu_{2}, \lambda_{2}, \lambda_{3}, \lambda_{4}, \lambda_{5}\right)
$$

It is interesting to trade three of the above parameters of the scalar sector for the physical masses of the new scalars through Eqs. (2.8)-(2.10). This will be important for the renormalization program when we adopt an on-shell scheme. ${ }^{2}$ The model can therefore be defined through the following two possible trade-offs,

$\left(\mu_{2}, \lambda_{3}, \lambda_{4}, \lambda_{5} ; \lambda_{2}\right) \rightarrow\left(M_{H}, M_{A}, M_{H^{ \pm}}, \lambda_{L / A} ; \lambda_{2}\right)$,

or equivalently

$$
\left(\mu_{2}, \lambda_{3}, \lambda_{4}, \lambda_{5} ; \lambda_{2}\right) \rightarrow\left(M_{H}, M_{A}, M_{H^{ \pm}}, \mu_{2} ; \lambda_{2}\right)
$$

We set $\lambda_{2}$ apart as it describes couplings solely between the additional scalars and not involving the SM Higgs. At tree level for example and for $2 \rightarrow 2$ annihilation processes, $\lambda_{2}$ is irrelevant. This would mean that at one-loop order, for annihilation processes, a renormalization for $\lambda_{2}$ is not necessary. However, $\lambda_{4}$ and $\lambda_{5}$ can be reconstructed from a combination of the additional scalar masses

$$
\begin{gathered}
\lambda_{4}=\frac{1}{v^{2}}\left(M_{H}^{2}+M_{A}^{2}-2 M_{H^{ \pm}}^{2}\right), \\
\lambda_{5}=\frac{1}{v^{2}}\left(M_{H}^{2}-M_{A}^{2}\right) .
\end{gathered}
$$

The extraction of $\lambda_{3}$ not only requires a knowledge of at least one scalar mass but also either a value of $\lambda_{L}$ (or equivalently the $h H H$ coupling) or the mass parameter $\mu_{2}$, to wit

$$
\lambda_{3}=\frac{2}{v^{2}}\left(M_{H^{ \pm}}^{2}-\mu_{2}^{2}\right)=\frac{2}{v^{2}}\left(M_{H^{ \pm}}^{2}-M_{H}^{2}\right)+\lambda_{L} .
$$

\section{RENORMALIZATION OF THE IDM}

The presence of the $\mathbb{Z}_{2}$ symmetry tremendously eases the renormalization of the IDM. As a result of this symmetry there is no mixing, at any order, between the SM fields and the extra fields introduced by the IDM. The tadpole condition only applies to the SM part. The SM part, including the SM Higgs (and the Goldstone bosons), are renormalized, independently and exactly as in the SM. We

\footnotetext{
${ }^{2}$ Note that $s_{W}$ was also defined in terms of the $W$ and $Z$ masses, Eq. (2.4).
} 
therefore follow an on-shell (OS) scheme whose details can be found in Ref. [27]. We will pursue the OS approach for all three extra physical scalar fields, $H, A, H^{ \pm}$. We will therefore use the physical masses of these fields as input parameters instead of the parameters of the scalar potential. Nonetheless, there remain two parameters which we still need to define. As stated earlier, of all the parameters in the IDM, only $\lambda_{2}$ connects the extra fields. Its renormalization is not needed for one-loop annihilation processes to SM particles. However, one parameter (either $\mu_{2}$ or $\lambda_{L, A}$ or a combination of these) is still needed to fully define all the couplings between $H, A, H^{ \pm}$and the SM Higgs and Goldstones; see Eq. (2.19). For example $\lambda_{L / A}$ has, at tree level, a simple physical interpretation as the portal coupling $h H H / h A A$.

To carry the renormalization program and define the counterterms, shifts are introduced for the Lagrangian parameters and the fields. All bare quantities $\left(X_{0}\right)$, particularly in Eq. (2.2), are decomposed into renormalized quantities $(X)$ and counterterms $(\delta X)$ as

$$
X_{0} \rightarrow X+\delta X, \quad X=\mu_{2}, \lambda_{2}, \lambda_{3}, \lambda_{4}, \lambda_{5},
$$

for the parameters ${ }^{3}$ and

$$
\phi_{0} \rightarrow \phi+\frac{1}{2} \delta Z_{\phi}, \quad \phi=\left(h, H, A, H^{ \pm}\right),
$$

for the fields.

The OS conditions on the physical scalars require that their masses are defined as pole masses of the renormalized one-loop propagator and that the residue at the pole be unity. With $\Sigma_{\phi \phi}\left(p^{2}\right)$ being the scalar two-point function with momentum $p$ we have $\left(\phi=h, H, A, H^{ \pm}\right)$,

$$
\begin{gathered}
\delta M_{\phi}^{2}=\Sigma_{\phi \phi}\left(M_{\phi}^{2}\right), \\
\delta Z_{\phi}=-\left.\frac{\partial \Sigma_{\phi \phi}\left(p^{2}\right)}{\partial p^{2}}\right|_{p^{2}=M_{\phi}^{2}} .
\end{gathered}
$$

$\delta M_{H}, \delta M_{A}$ and $\delta M_{H^{ \pm}}$directly give OS definitions for $\lambda_{4}$ and $\lambda_{5}$ through Eqs. (2.21) and (2.22). With only three physical masses, we cannot reconstruct all five counterterms from two-point functions. We therefore revert to couplings between the Higgses. Remembering that $\lambda_{L}$ measures the $h H H$ coupling, we could extract a counterterm for $\delta \lambda_{L}$ from a measurement of the $h H H$ coupling. We could have also chosen the $h A A$ or $h H^{+} H^{-}$couplings. Sticking with $\lambda_{L}$, when $M_{h}>2 M_{H}$, the invisible width of the Higgs $\Gamma(h \rightarrow H H)$ is the observable of choice especially because no infrared divergence affects this

\footnotetext{
${ }^{3}$ This procedure is also applied to the SM sector including the $\mu_{1}$ and $\lambda_{1}$ terms of potential. For the latter, the tadpole condition is imposed at one-loop; see [27].
}

observable. This observable will therefore be set as input, which is equivalent to stating that the observable receives no correction. We denote the amplitude for $h \rightarrow H H$ as $\mathcal{A}(h \rightarrow H H) \equiv \mathcal{A}_{h H H}$. We express this amplitude at tree level as

$$
\mathcal{A}_{h H H}^{0}=-\lambda_{L} v,
$$

and the full one-loop renormalized amplitude for $h(p) \rightarrow$ $H\left(p_{1}\right) H\left(p_{2}\right)$ as

$$
\begin{aligned}
\mathcal{A}_{h H H}^{\mathrm{ren}}\left(p^{2}, p_{1}^{2}, p_{2}^{2}\right)= & -\lambda_{L} v\left(\frac{\delta \lambda_{L}}{\lambda_{L}}+\frac{\delta v}{v}+\frac{1}{2} \delta Z_{h}+\delta Z_{H}\right) \\
& +\mathcal{A}_{H H h}^{1 \mathrm{PI}}\left(p^{2}, p_{1}^{2}, p_{2}^{2}\right),
\end{aligned}
$$

where $\mathcal{A}_{H H h}^{1 \mathrm{PI}}\left(p^{2}, p_{1}^{2}, p_{2}^{2}\right)$ is the full one-loop one particle irreducible vertex. When the threshold is open, we set $p^{2}=$ $M_{h}^{2}$ and $p_{1}^{2}=p_{2}^{2}=M_{H}^{2}$ defining a gauge invariant OS counterterm for $\lambda_{L}$ as

$\frac{\delta^{\mathrm{OS}} \lambda_{L}}{\lambda_{L}}=\frac{\mathcal{A}_{H H h}^{1 \mathrm{PI}}\left(m_{h}^{2}, M_{H}^{2}, M_{H}^{2}\right)}{\lambda_{L} v}-\frac{\delta v}{v}-\frac{1}{2} \delta Z_{h}-\delta Z_{H}$.

Another gauge invariant but scale-dependent scheme is to use an $\overline{\mathrm{MS}}$ definition where only the (mass-independent term) ultraviolet divergent part is kept

$\frac{\delta^{\overline{\mathrm{MS}}} \lambda_{L}}{\lambda_{L}}=\left(\frac{\mathcal{A}_{H H h}^{1 \mathrm{PI}}\left(m_{h}^{2}, M_{H}^{2}, M_{H}^{2}\right)}{\lambda_{L} v}-\frac{\delta v}{v}-\frac{1}{2} \delta Z_{h}-\delta Z_{H}\right)_{\infty}$.

The coefficient of the ultraviolet divergent part is nothing but the one-loop $\beta$ constant $\left(\beta_{\lambda_{L}}\right)$ of $\lambda_{L}$,

$$
\frac{\delta^{\overline{\mathrm{MS}}} \lambda_{L}}{\lambda_{L}}=\beta_{\lambda_{L}} C_{\mathrm{UV}}, \quad C_{\mathrm{UV}}=\frac{2}{\varepsilon}-\gamma_{E}+\ln (4 \pi),
$$

where $\varepsilon=4-d$ with $d$ being the number of dimensions in dimensional regularization and $\gamma_{E}$ being the Euler constant. As discussed at length in Ref. [28], a general scheme can be defined as

$$
\frac{\delta \lambda_{L}}{\lambda_{L}}=\beta_{\lambda_{L}}\left(C_{\mathrm{UV}}+\ln \left(\bar{\mu}^{2} / Q_{\lambda}^{2}\right)\right),
$$

where $Q_{\lambda}$ is an effective scale that depends on the external momenta (hence the subtraction point) and the internal masses introduced to define the counterterm, and $\bar{\mu}$ is the scale introduced by dimensional reduction. For the $\overline{\mathrm{MS}}$, scheme $Q_{\lambda}=\bar{\mu}$.

For $m_{h}<2 M_{H}$, it is difficult to come up with a straightforward OS scheme for $\lambda_{L}$ (or equivalently $\mu_{2}$ once the mass counterterms for the extra scalars have been set). 
We have therefore chosen an $\overline{\mathrm{MS}}$ scheme for $\lambda_{L}$ according to Eq. (3.9). A formal OS extraction that would work for any configuration of $H$ and $h$ masses could use the cross section that builds up direct detection, namely $H q \rightarrow H q$ in the limit of zero $Q^{2}$ transfer, in effect isolating the $H\left(M_{H}^{2}\right) \rightarrow H\left(M_{H}^{2}\right) h\left(Q^{2} \rightarrow 0\right)$ vertex. But direct detection involves uncertainties through the introduction of parameters from nuclear matrix elements. Moreover, the $\lambda_{L}$ contribution to direct detection can be swamped by the pure gauge contribution (which we discuss later).

As stated before, the counterterm for $\mu_{2}$ is directly related to that of $\lambda_{L}$ :

$$
\delta \mu_{2}^{2}=\delta M_{H}^{2}-\frac{v^{2}}{2} \delta \lambda_{L}-\lambda_{L} v^{2} \frac{\delta v}{v} .
$$

For the annihilation processes we will find that a counterterm for $\lambda_{2}$ is not necessary; however an $\overline{\mathrm{MS}}$ definition based on the corresponding $\beta$ function can be derived. This is shown in Appendix B.

\section{A HIGH MASS IDM BENCHMARK POINT}

Previous studies on the collider and astrophysical constraints of the IDM parameter space [6,14,26,29-51] have delineated two regions with a viable DM candidate that provides the correct relic density of DM. The first one is dubbed the "low mass regime" where $M_{H} \approx M_{h} / 2$ and the second the "high mass regime" where $M_{H} \gtrsim 500 \mathrm{GeV}$. To show the impact of a more precise calculation of the annihilation cross sections that enter the relic density, in this paper we start by finding a point that passes the relic density constraints based on a tree-level calculation. For this we use the code micrOMEGAs $[52,53]$. The relic density constraint set by Planck [21],

$$
\Omega h^{2}=0.1197 \pm 0.0022,
$$

is imposed.

The characteristics of our benchmark point are the following:

$$
\begin{aligned}
M_{H} & =550 \mathrm{GeV}, \quad M_{A}=551 \mathrm{GeV}, \quad M_{H}^{ \pm}=552 \mathrm{GeV}, \\
\lambda_{L} & =0.0193, \quad \lambda_{2}=0.01 \\
\left(\lambda_{3}\right. & =0.0926, \quad \lambda_{4}=-0.0545 \\
\lambda_{5} & \left.=-0.0181 \text { and } \mu_{2}=549.45 \mathrm{GeV}\right) .
\end{aligned}
$$

The values between brackets in Eq. (4.2) are derived values. For the SM parameters, we take $M_{h}=125 \mathrm{GeV}$, $M_{W}=80.45 \mathrm{GeV}, \quad M_{Z}=91.19 \mathrm{GeV}$ and $\alpha=1 / 137$. For these values of the parameters, the calculated relic density (calculated with tree-level cross sections) is $\Omega h^{2} \simeq 0.117$, a value consistent with Eq. (4.1). At tree level, the cross section does not depend on $\lambda_{2}$. Note that the viability of this point relies on the almost degenerate IDM scalar spectrum and the small values of the $\lambda$ 's. These small values of $\lambda$ 's automatically ensure that a perturbative calculation can be performed. Moreover, vacuum stability holds $[6,54]$ together with the fact that the global minimum is associated with the inert vacuum [9]. The degeneracy in the scalar masses can be viewed as rather fine-tuned [6]. This degeneracy means that constraints from electroweak precision measurements are easily evaded, in particular, the custodial isospin symmetry parameter $T$ [48]. Indeed in this case,

$$
\Delta T \simeq \frac{1}{24 \pi^{2} \alpha v^{2}}\left(M_{H^{ \pm}}-M_{A}\right)\left(M_{H^{ \pm}}-M_{H}\right)
$$

is vanishingly small.

Far more stringent is the constraint from the spinindependent DM-nucleon cross section for direct detection. In this scenario, the one-loop electroweak gauge contribution to the $H$ nucleon cross section, $\sigma_{H N}^{(g)}[17,55]$, is almost an order of magnitude larger than the tree-level Higgs exchange contribution triggered by $\lambda_{L}, \sigma_{H N}^{\left(\lambda_{L}\right)}$ [56]. One obtains

$$
\sigma_{H N}^{\left(\lambda_{L}\right)}=f^{2} \frac{\lambda_{L}^{2}}{4 \pi}\left(\frac{m_{N}^{2}}{m_{H} m_{h}^{2}}\right)^{2}
$$

where $m_{N}$ is the nucleon mass, and $f \sim 1 / 3$ is the nucleon form factor. With $M_{H} \gg M_{W}$, one can write

$$
\begin{aligned}
\mathcal{R}\left(\lambda_{L}\right) & =\frac{\sigma_{H N}^{(g)}}{\sigma_{H N}^{\left(\lambda_{L}\right)}} \sim\left(6 \pi \frac{\alpha^{2}}{\lambda_{L} s_{W}^{4}}\right)^{2}\left(\frac{M_{H}}{8 M_{W}}\right)^{2}\left(1+\frac{M_{h}^{2}}{M_{W}^{2}}\right)^{2} \\
& \sim 9 \text { for } \lambda_{L}=0.019 \text { and } M_{H}=550 \mathrm{GeV} .
\end{aligned}
$$

This benchmark point passes the present XENON1T [57] constraint. However, further improvement in the experimental sensitivity from direct detection experiments could make the viability of this benchmark point difficult even if the relic density constraint is passed.

The degeneracy in the scalar masses means that the relic density is built up by a few coannihilation channels. They mainly annihilate into vector bosons. The percentage contribution of each channel to the relic density is

$$
\left\{\begin{array}{l}
H H \rightarrow W^{+} W^{-}(18 \%), \\
H H \rightarrow Z Z(14 \%), \\
H^{+} H^{-} \rightarrow W^{+} W^{-}(13 \%), \\
A A \rightarrow W^{+} W^{-}(9 \%), \\
H^{+} H \rightarrow W^{+} \gamma(8 \%), \\
A A \rightarrow Z Z(7 \%), \\
H^{+} A \rightarrow W^{+} \gamma(6 \%) .
\end{array}\right.
$$


These weights are given by micrOMEGAs based on tree-level calculations. The other channels contribute less than or equal to $5 \%$, including the $h h$ final state channel.

Out of the seven cross sections, those with annihilations to a photon, $H^{+} A \rightarrow W^{+} \gamma$ and $H^{+} H \rightarrow W^{+} \gamma$ are driven solely by gauge couplings and are, at tree level, independent of $\lambda_{L, A}$. The other five interactions are sensitive to $\lambda_{L}$. However, considering the small value of $\lambda_{L}$ in our benchmark example, to a good approximation these cross sections are also dominantly (though not totally) driven by gauge interactions so we can write $\sigma_{H H \rightarrow W^{+} W^{-}} \sim$ $\sigma_{A A \rightarrow W^{+} W^{-}} \sim \sigma_{H^{+} H^{-} \rightarrow W^{+} W^{-}}=2 c_{W}^{4} \sigma_{H H \rightarrow Z Z}=2 c_{W}^{4} \sigma_{A A \rightarrow Z Z}$. The weights quoted in Eq. (4.6) are a measure of the relative importance of the corresponding cross sections diluted by the Boltzmann factor. We will now look at the one-loop corrections that affect the seven dominant processes given in Eq. (4.6) which, at tree level, contribute more than $5 \%$ to the relic density. We will compute these processes for a wide range of velocities.

\section{ANNIHILATION CROSS SECTIONS AT ONE-LOOP ORDER}

\section{A. Some important technicalities}

The calculation of the relic density requires the dependence of the different relevant cross sections on the relative velocity $v$ of the annihilating particles times $v, \sigma_{i j} v_{i j}$, where $i, j$ stand for the annihilating/coannihilating particles, before applying thermal averaging. Within the standard cosmological model and assuming freeze-out, the latter part is computed quite precisely by micrOMEGAs. For two annihilating particles with momenta $p_{1}$ and $p_{2}$ and masses $m_{1}$ and $m_{2}$, the relative velocity is defined as

$$
\begin{aligned}
& v=2 s \frac{\sqrt{\left(s-\left(m_{1}+m_{2}\right)^{2}\right)\left(s-\left(m_{1}-m_{2}\right)^{2}\right)}}{s^{2}-\left(m_{1}^{2}-m_{2}^{2}\right)^{2}}, \quad s=\left(p_{1}+p_{2}\right)^{2}, \\
& v=2 \sqrt{1-4 M_{\mathrm{DM}}^{2} / s}=2 \beta \text { for } m_{1}=m_{2}=M_{\mathrm{DM}} .
\end{aligned}
$$

However, it is possible to replace the tree-level cross sections generated by micrOMEGAs by corrected cross sections. This is what we do in order to obtain loop-corrected relic density. In our case, the corrected cross sections are the one-loop-corrected cross sections for the processes listed in Eq. (4.6). Most of the steps of the calculation are automated. We rely on Sloops [58-61] which relies on LanHEP $[62,63]$ to define the model. LanHEP generates the complete set of Feynman rules, applying shifts on fields and parameters and sets the conditions on the generated counterterms in a format compatible with an amplitude generator code. We interface LanHEP with the package bundle FeynArts, FormCalc and LoopTools [64-66].

For each process and for each velocity we check that the virtual corrections (including the counterterms) are ultraviolet finite. To this end we vary the $C_{\mathrm{UV}}$ [Eq. (3.9)] parameter by 7 orders of magnitude and check that the result is stable within machine (double) precision. For processes involving charged particles, bremsstrahlung processes $2 \rightarrow 2+\gamma$ are generated. The latter is split into two parts, the soft photon radiation and the hard photon radiation. The soft photon radiation for photon energies $E_{\gamma}<k_{c}$ with $k_{c}$ small enough is generated automatically through the factorization formula which eliminates the oneloop infrared divergence that we regularize with a small finite photon mass. The hard photon radiation is computed numerically. We loop over a few values of $k_{c}$ making sure that the soft plus hard part adds to a value that is insensitive to $k_{c}$. This step could be time consuming but we have optimized its automation. When we refer to next-toleading order corrections, we have in mind the full oneloop, the soft and the hard radiation which is of course independent of the regularizing photon mass or the intermediate cutoff $k_{c}$.

\section{B. Processes at one-loop}

Our default values for the one-loop corrections are presented in this subsection for a scale $\bar{\mu}=M_{H}$ taken to define $\lambda_{L}$. The scale dependence will be discussed when we convert the results to the level of the relic density calculation.

\section{1. $\mathrm{H}^{+} \mathrm{H}^{-} \rightarrow W^{+} W^{-}$}

We start our discussion with a process whose weight to the relic density (at tree level) is 13\%. Even though this is not the most dominant contribution, it helps bring forth an important behavior. At tree level, this process slowly and linearly varies with the relative velocity. This is still the case at one-loop where the full one-loop computation corrects the tree-level result by about $-10 \%$ for relative velocities $\sim 0.2$ and above. However, the one-loop contribution shoots with large positive "corrections" up to extremely low velocities; see Fig. 1. This is easily understood as a result of the electromagnetic Sommerfeld effect. The photon exchange between the electrically charged coannihilating particles at very low relative velocities leads to a relative correction which at one-loop reads as

$$
\frac{\Delta \sigma^{1 \text {-loop Somm }} v}{\sigma^{\text {tree }} v}=\frac{\pi \alpha}{v} .
$$

We have checked that our numerical code captures this effect exactly. This one-loop Sommerfeld contribution can be resummed with the result that the tree-level cross section is turned into

$$
\sigma^{\text {resummed }}=S_{\mathrm{nr}} \sigma^{\text {tree }}, \quad S_{\mathrm{nr}}=\frac{X_{\mathrm{nr}}}{1-e^{-X_{\mathrm{nr}}}} \text { and } X_{\mathrm{nr}}=2 \pi \alpha / v
$$



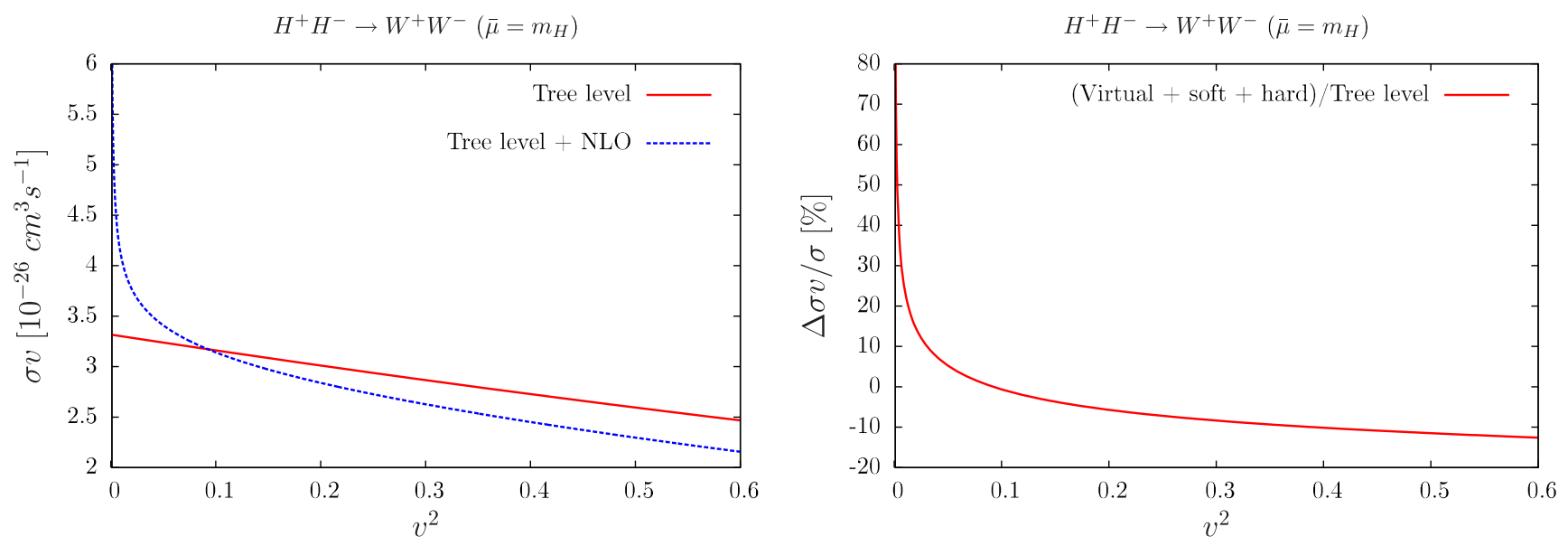

FIG. 1. Dependence of the tree-level and one-loop-corrected cross-section $H^{+} H^{-} \rightarrow W^{+} W^{-}$with respect to the relative velocity (squared). The right panel gives the percentage correction.

Since characteristic velocities for the calculation of the relic density are typically in the range $v \sim 0.2-0.3$, the Sommerfeld enhancement taken either at one-loop or resummed to all orders does not have much of an impact on the relic density. We have checked this feature explicitly. It is however important that our full calculation catches such behavior at very small velocities quite precisely.
While presenting our results for the relic density, this resummation is performed even though its effect is tiny.

Note that an electroweak equivalent to the Sommerfeld correction is induced by rescattering through $W$ and $Z$ bosons and even through the SM Higgs boson. For $H^{+} H^{-} \rightarrow W^{+} W^{-}$these low velocity effects are completely swamped by the photon exchange (Sommerfeld effect). For
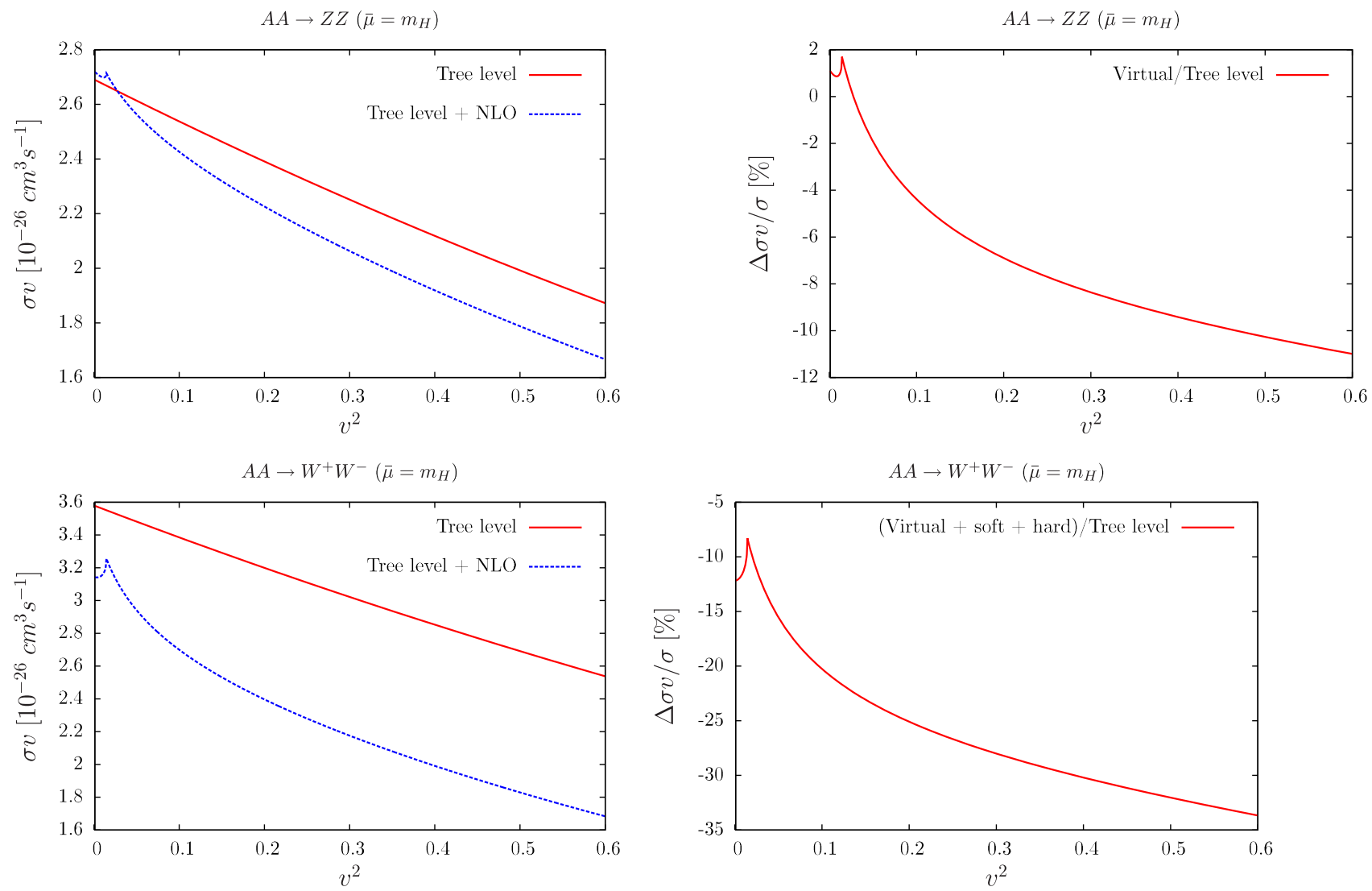

FIG. 2. Dependence of the tree-level and one-loop-corrected cross section $A A \rightarrow Z Z$ (upper panels) and $A A \rightarrow W^{+} W^{-}$with respect to the relative velocity (squared). The right panels give the percentage correction. 

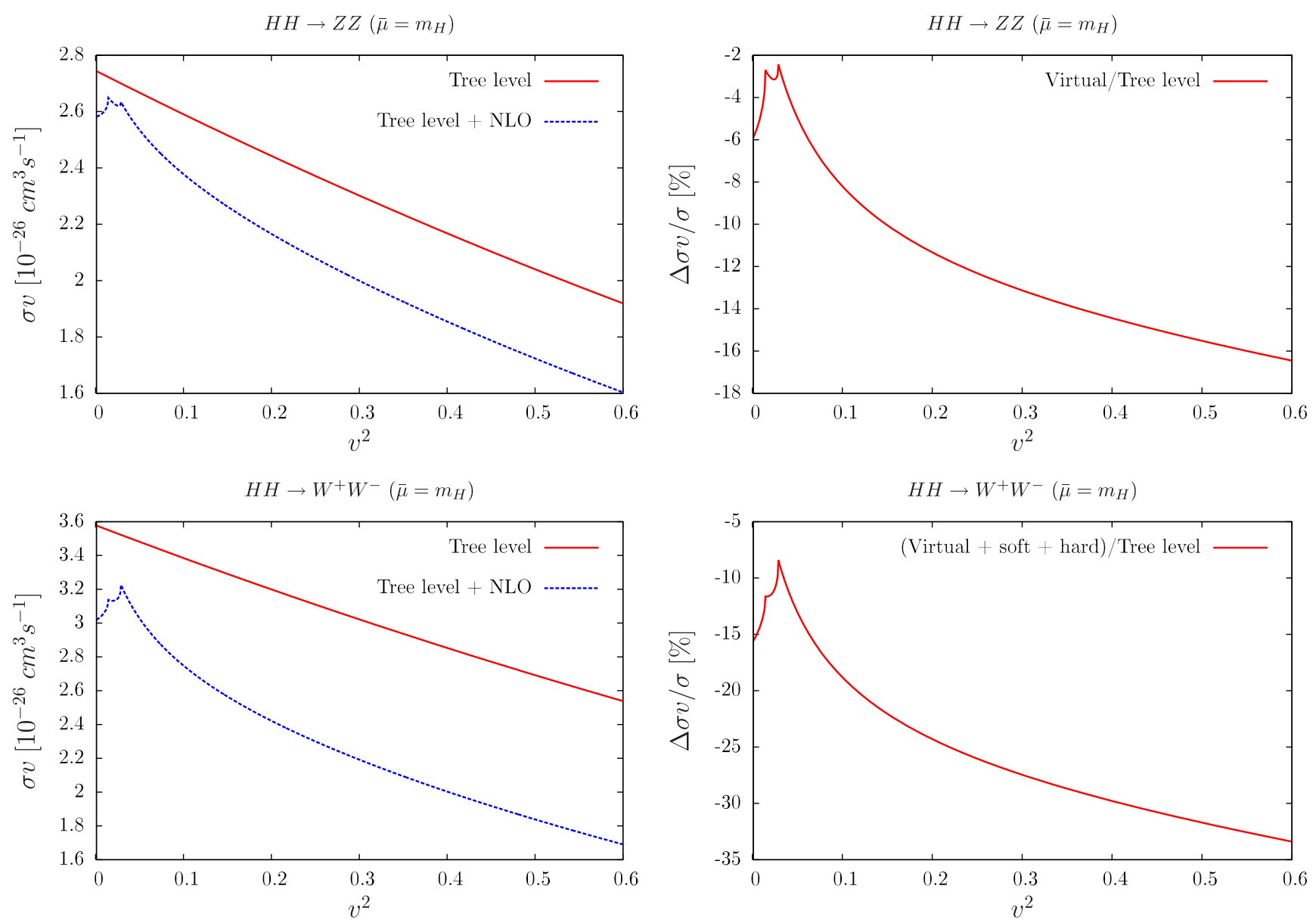

FIG. 3. As in Fig. 2 but for $H H \rightarrow Z Z$ and $H H \rightarrow W^{+} W^{-}$.

later reference, let us point out that these electroweak equivalents may play a role at very small velocities only if $M_{W, Z, h} / M_{\mathrm{DM}} \ll 1$, which is not attained in our scenario. The masses of the $W, Z$ and $h$ bosons provide a cutoff to the $1 / v$ rise, so the rise of the cross section, when the massive bosons are involved, is not indefinite. Higgs exchange will be totally negligible considering that the $h H H$ coupling, for example, is controlled by the small $\lambda_{L}$.

\section{2. $A A \rightarrow Z Z$ and $A A \rightarrow W^{+} W^{-}$}

$A A \rightarrow Z Z$ and $A A \rightarrow W^{+} W^{-}$contribute respectively $7 \%$ and $10 \%$ to the relic density at tree level. The velocity dependence of their cross sections at tree level decreases slowly with an almost similar rate. At one-loop, the photon final state radiation affects the $W^{+} W^{-}$channel. The overall corrections are thus larger in the charged channel than in the neutral channel. For $v=0.3$ this correction is a modest $-5 \%$ in the $Z Z$ channel but about $-20 \%$ in the $W^{+} W^{-}$ channel. Nonetheless, the corrections follow a similar trend; see Fig. 2. For large velocities, the corrections are largest (and negative) and tend to decrease in absolute values by a contribution that behaves as $1 / v^{2}$ up to $v \sim 0.12$ where it again drops. This behavior at such small values of the relative velocity is a reminder of the Sommerfeld effect due to the $W$ exchange. In these two processes such effects are only triggered by $W$ exchange (and not by $Z$ exchange) since no $A A Z$ coupling exists, where $A H^{ \pm} W^{\mp}$ is operative. This rescattering, $A A \rightarrow H^{+} H^{-}$, also explains why the corrections in the $W^{+} W^{-}$channel are larger; indeed the amplitude for $H^{+} H^{-} \rightarrow W^{+} W^{-}$is more than twice as large as the $\mathrm{H}^{+} \mathrm{H}^{-} \rightarrow \mathrm{ZZ}$ counterpart.

\section{3. $\mathrm{HH} \rightarrow \mathrm{ZZ}$ and $\mathrm{HH} \rightarrow \mathrm{W}^{+} W^{-}$}

As expected, for small $\lambda$ 's, the $H \leftrightarrow A$ have the same cross section at tree level; see Fig. 3. Their dependence on velocity is the same, as are the radiative corrections apart from a notable difference for very small velocities. The $H H$ annihilations with respect to the relative velocity now feature two dents, contrary to the $A A$ annihilations where one dent appears. The first of these dents occurs at practically the same location in $v$ as the one that occurs for the $A A$ annihilations. It corresponds to the exchange of the $W$. The second one at slightly larger velocities is due to the $Z$ exchange. Again the corresponding velocities are too small to be relevant for the calculation of the relic density. 

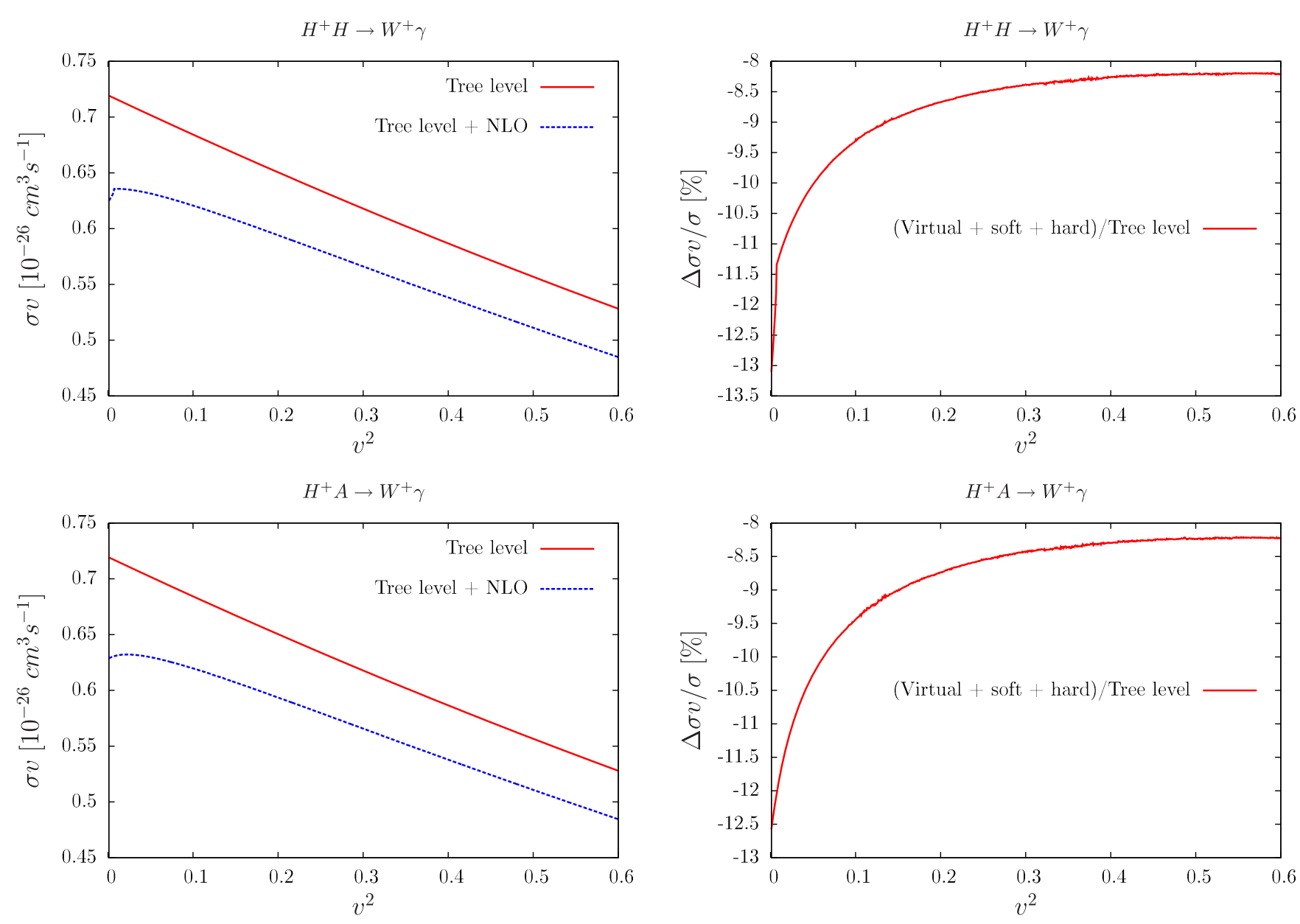

FIG. 4. As in Fig. 3 but for $H^{+} H \rightarrow W^{+} \gamma$ and $H^{+} A \rightarrow W^{+} \gamma$. The one-loop corrections are $\bar{\mu}$ independent since they are totally driven by the gauge interactions.

\section{4. $\mathrm{H}^{+} \mathrm{H} \rightarrow \mathrm{W}^{+} \gamma$ and $\mathrm{H}^{+} \mathrm{A} \rightarrow \boldsymbol{W}^{+} \gamma$}

Again these two cross sections are practically interchangeable both at the leading and at next-to-leading order. The relative one-loop corrections decrease as the relative velocity decreases, with a correction of about $-10 \%$ for a typical velocity, $v \sim 0.3$; see Fig. 4 . Since at tree level these processes do not depend on $\lambda_{L, A}$, a fully on-shell renormalization is possible with the results that these one-loop cross sections are $\bar{\mu}$ independent.

\section{RELIC ABUNDANCE COMPUTATIONS}

Having performed the full one-loop corrections to the seven processes that make up about $70 \%$ of the total relic density at tree level, we have interfaced our calculations with micrOMEGAs by providing the tables for these cross sections (with the velocity dependence) in lieu of their treelevel value to micrOMEGAs for the calculation of the relic density (thermal averaging, freeze-out). The remaining processes $\left(H^{+} H^{-} \rightarrow \gamma \gamma, \quad H^{+} H^{-} \rightarrow \gamma Z, H^{+} H \rightarrow Z W^{+}\right.$, $H^{+} A \rightarrow Z W^{+}, H^{+} H^{-} \rightarrow Z Z$ and $\left.H^{+} H^{-} \rightarrow h h\right)$ were kept at their tree-level values. For the loop-corrected
$H^{+} H^{-} \rightarrow W^{+} W^{-}$we compared the result of the relic density with full one-loop calculation, the subtraction of the Sommerfeld correction [Eq. (5.2)], and the replacement of the Sommerfeld contribution with its resummed classical result [Eq. (5.3)]. As expected, we found no noticeable change between the different implementation of the QED Sommerfeld effect. As we saw above, all the cross sections we calculated at one-loop are affected by a negative correction for $\bar{\mu}=M_{H}$. Here we recall that we had taken as input $\alpha=\alpha(0)$; the sign and size of the corrections are not due to the running of $\alpha$. Smaller one-loop cross sections compared to tree level translate to a larger relic density than derived from tree-level cross sections. This is corroborated by the value $\Omega h^{2}=0.12494$ that we find. For $\bar{\mu}=M_{H} / 2$, the loop corrections are smaller, and this naturally translates into a smaller correction to the relic density. Indeed we find a correction of only $\sim-0.3 \%$. This would tend to suggest that this scale, for the aforementioned choice of parameters, is optimal for reducing the size of the radiative corrections. For $\mu=2 M_{H}$, the correction to the relic density amounts to $\sim 15.3 \%$. The scale variation is large compared to the experimental precision on the relic density. Considering 
TABLE I. Relative contributions to the relic abundance with and without corrections. Note that although the cross sections for the last five processes (identified with $\bullet$ ) are not loop corrected, their relative contribution could change.

\begin{tabular}{lrccc}
\hline \hline Process & LO & $\mu=m_{X}$ & $\mu=m_{X} / 2$ & $\mu=2 \times m_{X}$ \\
\hline$H H \rightarrow W^{+} W^{-}$ & $18 \%$ & $16 \%$ & $18 \%$ & $14 \%$ \\
$H H \rightarrow Z Z$ & $14 \%$ & $14 \%$ & $14 \%$ & $14 \%$ \\
$H^{+} H^{-} \rightarrow W^{+} W^{-}$ & $13 \%$ & $15 \%$ & $15 \%$ & $15 \%$ \\
$A A \rightarrow W^{+} W^{-}$ & $9 \%$ & $8 \%$ & $9 \%$ & $7 \%$ \\
$H^{+} H \rightarrow W^{+} \gamma$ & $8 \%$ & $7 \%$ & $7 \%$ & $8 \%$ \\
$A A \rightarrow Z Z$ & $7 \%$ & $7 \%$ & $7 \%$ & $8 \%$ \\
$H^{+} A \rightarrow W^{+} \gamma$ & $6 \%$ & $6 \%$ & $6 \%$ & $7 \%$ \\
$\bullet H^{+} H^{-} \rightarrow \gamma \gamma$ & $5 \%$ & $5 \%$ & $5 \%$ & $6 \%$ \\
$\bullet H^{+} H^{-} \rightarrow \gamma Z$ & $4 \%$ & $5 \%$ & $4 \%$ & $5 \%$ \\
$\cdot H^{+} H \rightarrow Z W^{+}$ & $3 \%$ & $3 \%$ & $3 \%$ & $3 \%$ \\
$\cdot H^{+} A \rightarrow Z W^{+}$ & $3 \%$ & $3 \%$ & $2 \%$ & $3 \%$ \\
$\bullet H^{+} H^{-} \rightarrow Z Z$ & $2 \%$ & $2 \%$ & $2 \%$ & $2 \%$ \\
\hline \hline
\end{tabular}

that these scenarios are quite fine-tuned, for an almost degenerate scalar mass spectrum, our calculations show that if one is performing a tree-level analysis one should not strictly impose the very constrained experimental bound on the relic density but one should allow a theoretical uncertainty of at least about $10 \%$ for such benchmark scenarios. We may ask whether the radiative corrections change much the relative weights of the different processes. Table I shows that these relative contributions experience little change, independent of the scale chosen. The most important change is therefore an almost uniform correction on all the cross sections.

\section{NEWS FROM THE DARK SECTOR: IMPACT OF $\lambda_{2}$}

$\lambda_{2}$ does not enter the calculation of the annihilation cross sections at tree level. But, just as the $Z$ decay to muons does not depend on the top quark mass at tree level, at one-loop the top quark makes its effect felt. For the case at hand, there is less subtlety for the nondecoupling of the self-coupling in the dark sector. $H H$ can rescatter before annihilating to gauge bosons. The rescattering $H H \rightarrow H H$ involves the self-coupling $\lambda_{2}$. One therefore expects the one-loop annihilating cross sections to depend on $\lambda_{2}$. To investigate this effect, we retain the same value of $\lambda_{L}$ and consider two other values of $\lambda_{2}$, both well within the perturbative and positivity of the potential bound, $\lambda_{2}=0.1$ and $\lambda_{2}=1$. Our results are shown in Table II. We observe that there is a noticeable albeit small change when $\lambda_{2}$ is increased to 0.1 , the scale variation is reduced and the total electroweak corrections to the relic density are below $7.8 \%$. The case $\lambda_{2}=1$ is much more interesting. The corrections are now quite large for each of the three renormalization scales $M_{H} / 2, M_{H}$ and $2 M_{H}$. For all of these three scales, the tree-level benchmark point would be ruled out. However, we note that the large-scale uncertainty with corrections ranging between $+21.2 \%$ for
TABLE II. Dependence of the relic density on the parameter $\lambda_{2}$ and the influence of the scale variation. The percentage change is shown within brackets.

\begin{tabular}{llll}
\hline \hline$\lambda_{2}$ & \multicolumn{1}{c}{$\mu=M_{H}$} & \multicolumn{1}{c}{$\mu=M_{H} / 2$} & \multicolumn{1}{c}{$\mu=2 M_{H}$} \\
\hline 0.01 & $0.12494(6.9 \%)$ & $0.11652(-0.3 \%)$ & $0.13469(15.3 \%)$ \\
0.1 & $0.12210(4.5 \%)$ & $0.11843(1.3 \%)$ & $0.12601(7.8 \%)$ \\
1 & $0.09950(-14.9 \%)$ & $0.14163(21.2 \%)$ & $0.07683(-34.3 \%)$ \\
\hline \hline
\end{tabular}

$\mu=M_{h} / 2$ and $-34.3 \%$ for $\mu=2 M_{H}$ means that a judicious scale choice, within the range $M_{H} / 2$ to $2 M_{H}$, can minimize the corrections. A more thorough one-loop analysis is in order by studying other scenarios with a larger range of values for the other quartic couplings. One could find points not allowed by a tree-level analysis that could be validated by a one-loop analysis. We leave this interesting analysis for a future publication. It looks however that although the virtual effect of $\lambda_{2}$ is not at all negligible it (fortunately or unfortunately) introduces also a non-negligible scale variation to the corrections. More importantly, compared to a treelevel treatment, one-loop corrections introduce not only a scale uncertainty which is manageable for small values of $\lambda_{2}$ but also a parametric dependence (dependence on $\lambda_{2}$ ) which is not caught by a tree-level treatment. In fact, this $\lambda_{2}$ dependence turns out to be even larger than the scale dependence. One could in fact use the measurement of the relic density to constrain $\lambda_{2}$.

\section{CONCLUSIONS}

The experimental value of the relic density as extracted from Planck data is now at the percent level. For many particle physics models of dark matter this is a very stringent bound that reduces drastically the range of the parameters in that model. Assuming a standard cosmological model based on freeze-out, the restriction on the parameter space of the model arises from the contribution of the annihilation and coannihilation cross sections that build up the evaluation of the relic density. Unfortunately, most analyses are based on tree-level evaluations of these cross sections. The level of precision on the experimental bound on the relic density calls for a theoretical prediction that should go beyond a tree-level evaluation of these cross sections. Such a program has been set up for the minimal supersymmetric model [58-61,67-69] and the next-tominimal supersymmetric model [28]. After a first exploratory investigation in Ref. [70], the present paper has extended this program to the IDM. As such this paper has presented a full systematic renormalization of the model and specialized in the first application into the so-called heavy mass scenario. In fact, in order to be fully perturbative, here we have covered a scenario with heavy scalar masses not heavier than $550 \mathrm{GeV}$. We have performed full one-loop calculations to seven annihilation/coannihilation processes. We have interfaced these corrected cross sections with micrOMEGAs to turn these cross sections into a 
more precise evaluation of the relic density assuming the standard freeze-out mechanism. The one-loop calculations have been implemented in an automated code for loop calculations. We have used a mixed scheme where most of the parameters are defined on shell based on the physical masses of the model. Having exhausted all masses in the model to fully define the model, one parameter has been defined in $\overline{\mathrm{MS}}$, the coupling of the scalar DM, $H$, to the SM Higgs. We have found that the one-loop corrections to the relic density for this particular mass vary from $-34 \%$ to $+15 \%$ depending on the renormalization scale chosen to define the $h-H-H$ coupling and most importantly on the value of the coupling $\lambda_{2}$ which measures the interaction solely within the dark sector between the extra scalars. This is an indirect effect that should be taken into account especially for $\lambda_{2}$ of order 1 . Its effects can be larger than the renormalization scale uncertainty of the one-loop calculation, else the relic density calculation can be used to set a limit on the dark-sector interaction. A tree-level calculation of the relic density is totally insensitive to the couplings that describe interaction within the dark sector. Preliminary investigations for DM masses beyond $750 \mathrm{GeV}$ have shown that electroweak Sommerfeld effects become important and that some resummation needs to be performed and merged with perturbative purely one-loop effects like those triggered by rescattering in the dark sector (the indirect effects of $\lambda_{2}$ ). We leave the study of this mass range to a forthcoming publication. Note that a for masses beyond $1 \mathrm{TeV}$, a purely nonperturbative treatment has been given in $[5,25]$. Other viable parameter space of the IDM is the low mass regime with $M_{H} \approx M_{h} / 2$. This requires the calculation of $2 \rightarrow 3$ processes at one-loop for the evaluation of the relic density. We also leave this application for a forthcoming publication.

\section{ACKNOWLEDGMENTS}

We thank Alexander Pukhov for several helpful discussions at various stages of this work. The work of S. B. is supported by a Durham Junior Research Fellowship COFUNDed by Durham University and the European Union, under Grant Agreement No. 609412. N. C. acknowledges financial support from National Center for Theoretical Sciences and also thanks LAPTh for hospitality during the formative stages of the project. H. S. is supported by the National Natural Science Foundation of China (Grant No. 11675033) and by the Fundamental Research Funds for the Central Universities (Grant No. DUT18LK27). This work was initiated within CNRS Laboratoire International Associé Theoretical High Energy Physics and the IndoFrench Network on High Energy Physics of CEFIPRA Indo-French Centre for the Promotion of Advanced Research. S. B. and H. S. also thank LAPTh for hospitality where a part of this work was carried out. S. B. and F. B. acknowledge the Les Houches workshop series "Physics at TeV colliders" where this work was finalized.

\section{APPENDIX A: FEYNMAN RULES}

We give below the Feynman rules of the trilinear and quadrilinear couplings among the scalars of the model using the parametrization of Eq. (2.19).

\section{Cubic Higgs couplings}

$$
\begin{gathered}
h-h-h:-3 i\left(\frac{M_{h}^{2}}{v}-\frac{T}{v^{2}}\right), \\
h-H-H:-2 i\left(\frac{M_{H}^{2}-\mu_{2}^{2}}{v}\right)=-i \lambda_{L} v, \\
h-A-A:-2 i\left(\frac{M_{A}^{2}-\mu_{2}^{2}}{v}\right)=-i \lambda_{A} v, \\
h-H^{+}-H^{-}:-2 i\left(\frac{M_{H^{ \pm}}^{2}-\mu_{2}^{2}}{v}\right)=-i \lambda_{3} v .
\end{gathered}
$$

\section{Quartic Higgs couplings}

$$
\begin{gathered}
h-h-h-h:-3 i\left(\frac{M_{h}^{2}}{v^{2}}-\frac{T}{v^{3}}\right), \\
h-h-H-H:-2 i\left(\frac{M_{H}^{2}-\mu_{2}^{2}}{v^{2}}\right)=-i \lambda_{L}, \\
h-h-A-A:-2 i\left(\frac{M_{A}^{2}-\mu_{2}^{2}}{v^{2}}\right)=-i \lambda_{A}, \\
h-h-H^{+}-H^{-}:-2 i\left(\frac{M_{H^{ \pm}}^{2}-\mu_{2}^{2}}{v^{2}}\right)=-i \lambda_{3}, \\
H-H-H-H:-6 i \lambda_{2}, \\
A-A-A-A:-6 i \lambda_{2}, \\
H-H-A-A:-2 i \lambda_{2}, \\
H-H-H^{+}-H^{-}:-2 i \lambda_{2}, \\
A-A-H^{+}-H^{-}:-2 i \lambda_{2}, \\
H^{+}-H^{-}-H^{+}-H^{-}:-4 i \lambda_{2} .
\end{gathered}
$$




\section{APPENDIX B: COUNTERTERM FOR $\lambda_{2}$}

The $\overline{\mathrm{MS}}$ counterterm for $\lambda_{2}$ can be obtained from adapting the beta functions of the two Higgs doublet model to the IDM given in Ref. [71] for example. In particular, we can write

$$
\delta \lambda_{2}^{\overline{\mathrm{MS}}}=\frac{1}{32 \pi^{2}}\left(\hat{\beta}_{\lambda_{2}}^{S}+\hat{\beta}_{\lambda_{2}}^{g}\right) C_{\mathrm{UV}},
$$

with

$$
\begin{gathered}
\hat{\beta}_{\lambda_{2}}^{S}=24 \lambda_{2}^{2}+2 \lambda_{3}^{2}+2 \lambda_{3} \lambda_{4}+\lambda_{4}^{2}+\lambda_{5}^{2}, \\
\hat{\beta}_{\lambda_{2}}^{g}=\frac{3}{8}\left(3 g^{4}+g^{\prime 4}+2 g^{2} g^{\prime 2}-3 \lambda_{2}\left(3 g^{2}+g^{\prime 2}\right)\right),
\end{gathered}
$$

where $g=e / s_{W}, g^{\prime}=e / c_{W}$.
[1] N. G. Deshpande and E. Ma, Pattern of symmetry breaking with two Higgs doublets, Phys. Rev. D 18, 2574 (1978).

[2] R. Barbieri, L. J. Hall, and V.S. Rychkov, Improved naturalness with a heavy Higgs: An alternative road to LHC physics, Phys. Rev. D 74, 015007 (2006).

[3] T. Hambye and M. H. G. Tytgat, Electroweak symmetry breaking induced by dark matter, Phys. Lett. B 659, 651 (2008).

[4] Q.-H. Cao, E. Ma, and G. Rajasekaran, Observing the dark scalar doublet and its impact on the standard-model Higgs boson at colliders, Phys. Rev. D 76, 095011 (2007).

[5] T. Hambye, F. S. Ling, L. L. Honorez, and J. Rocher, Scalar multiplet sark matter, J. High Energy Phys. 07 (2009) 090; Erratum: scalar multiplet dark matter, J. High Energy Phys.05 (2010) 66.

[6] A. Goudelis, B. Herrmann, and O. Stål, Dark matter in the inert doublet model after the discovery of a Higgs-like boson at the LHC, J. High Energy Phys. 09 (2013) 106.

[7] A. Arhrib, Y.-L. Sming Tsai, Q. Yuan, and T.-C. Yuan, An updated analysis of inert Higgs doublet model in light of the recent results from LUX, PLANCK, AMS-02 and LHC, J. Cosmol. Astropart. Phys. 06 (2014) 030.

[8] F. S. Queiroz and C.E. Yaguna, The CTA aims at the inert doublet model, J. Cosmol. Astropart. Phys. 02 (2016) 038.

[9] A. Belyaev, G. Cacciapaglia, I. P. Ivanov, F. Rojas-Abatte, and M. Thomas, Anatomy of the inert two Higgs doublet model in the light of the LHC and non-LHC dark matter searches, Phys. Rev. D 97, 035011 (2018).

[10] G. Arcadi, A. Djouadi, and M. Raidal, Dark matter through the Higgs portal, arXiv:1903.03616.

[11] S. Kanemura, S. Kiyoura, Y. Okada, E. Senaha, and C. P. Yuan, New physics effect on the Higgs self-coupling, Phys. Lett. B 558, 157 (2003).

[12] E. Senaha, Radiative corrections to triple Higgs coupling and electroweak phase transition: Beyond one-loop analysis, Phys. Rev. D 100, 055034 (2019).

[13] J. Braathen and S. Kanemura, On two-loop corrections to the Higgs trilinear coupling in models with extended scalar sectors, Phys. Lett. B 796, 38 (2019).

[14] A. Arhrib, R. Benbrik, J. El Falaki, and A. Jueid, Radiative corrections to the triple Higgs coupling in the inert Higgs doublet model, J. High Energy Phys. 12 (2015) 007.
[15] P. M. Ferreira and B. Swiezewska, One-loop contributions to neutral minima in the inert doublet model, J. High Energy Phys. 04 (2016) 099.

[16] P. M. Ferreira and D. R. T. Jones, Bounds on scalar masses in two Higgs doublet models, J. High Energy Phys. 08 (2009) 069.

[17] M. Klasen, C. E. Yaguna, and J. D. Ruiz-Alvarez, Electroweak corrections to the direct detection cross section of inert Higgs dark matter, Phys. Rev. D 87, 075025 (2013).

[18] A. Arhrib, R. Benbrik, and N. Gaur, $H \rightarrow \gamma \gamma$ in inert Higgs doublet model, Phys. Rev. D 85, 095021 (2012).

[19] M. Gustafsson, E. Lundstrom, L. Bergstrom, and J. Edsjo, Significant Gamma Lines from Inert Higgs Dark Matter, Phys. Rev. Lett. 99, 041301 (2007).

[20] C. Garcia-Cely, M. Gustafsson, and A. Ibarra, Probing the inert doublet dark matter model with Cherenkov telescopes, J. Cosmol. Astropart. Phys. 02 (2016) 043.

[21] P. A. R. Ade et al., Planck 2015 results. XIII. Cosmological parameters, Astron. Astrophys. 594, A13 (2016).

[22] J. Hisano, S. Matsumoto, and M. M. Nojiri, Unitarity and higher order corrections in neutralino dark matter annihilation into two photons, Phys. Rev. D 67, 075014 (2003).

[23] J. Hisano, S. Matsumoto, and M. M. Nojiri, Explosive Dark Matter Annihilation, Phys. Rev. Lett. 92, 031303 (2004).

[24] J. Hisano, S. Matsumoto, M. M. Nojiri, and O. Saito, Nonperturbative effect on dark matter annihilation and gamma ray signature from galactic center, Phys. Rev. D 71, 063528 (2005).

[25] S. Biondini and M. Laine, Re-derived overclosure bound for the inert doublet model, J. High Energy Phys. 08 (2017) 047.

[26] A. Ilnicka, M. Krawczyk, and T. Robens, Inert doublet model in light of LHC Run I and astrophysical data, Phys. Rev. D 93, 055026 (2016).

[27] G. Belanger, F. Boudjema, J. Fujimoto, T. Ishikawa, T. Kaneko, K. Kato, and Y. Shimizu, Automatic calculations in high energy physics and GRACE at one-loop, Phys. Rep. 430, 117 (2006).

[28] G. Bélanger, V. Bizouard, F. Boudjema, and G. Chalons, One-loop renormalization of the NMSSM in SloopS. II. The Higgs sector, Phys. Rev. D 96, 015040 (2017).

[29] L. L. Honorez, E. Nezri, J. F. Oliver, and M. H. G. Tytgat, The inert doublet model: An archetype for dark matter, J. Cosmol. Astropart. Phys. 02 (2007) 028. 
[30] P. Agrawal, E. M. Dolle, and C. A. Krenke, Signals of inert doublet dark matter in neutrino telescopes, Phys. Rev. D 79, 015015 (2009).

[31] E. Lundstrom, M. Gustafsson, and J. Edsjo, The inert doublet model and LEP II limits, Phys. Rev. D 79, 035013 (2009).

[32] S. Andreas, M. H. G. Tytgat, and Q. Swillens, Neutrinos from inert doublet dark matter, J. Cosmol. Astropart. Phys. 04 (2009) 004.

[33] C. Arina, F.-S. Ling, and M. H. G. Tytgat, IDM and iDM or the inert doublet model and inelastic dark matter, J. Cosmol. Astropart. Phys. 10 (2009) 018.

[34] E. Dolle, X. Miao, S. Su, and B. Thomas, Dilepton signals in the inert doublet model, Phys. Rev. D 81, 035003 (2010).

[35] E. Nezri, M. H. G. Tytgat, and G. Vertongen, $e+$ and anti- $p$ from inert doublet model dark matter, J. Cosmol. Astropart. Phys. 04 (2009) 014.

[36] X. Miao, S. Su, and B. Thomas, Trilepton signals in the inert doublet model, Phys. Rev. D 82, 035009 (2010).

[37] J.-O. Gong, H. M. Lee, and S. K. Kang, Inflation and dark matter in two Higgs doublet models, J. High Energy Phys. 04 (2012) 128.

[38] M. Gustafsson, S. Rydbeck, L. Lopez-Honorez, and E. Lundstrom, Status of the inert doublet model and the role of multileptons at the LHC, Phys. Rev. D 86, 075019 (2012).

[39] B. Swiezewska and M. Krawczyk, Diphoton rate in the inert doublet model with a $125 \mathrm{GeV}$ Higgs boson, Phys. Rev. D 88, 035019 (2013).

[40] L. Wang and X.-F. Han, LHC diphoton Higgs signal and top quark forward-backward asymmetry in quasiinert Higgs doublet model, J. High Energy Phys. 05 (2012) 088.

[41] M. Krawczyk, D. Sokolowska, P. Swaczyna, and B. Swiezewska, Constraining inert dark matter by $R_{\gamma \gamma}$ and WMAP data, J. High Energy Phys. 09 (2013) 055.

[42] P. Osland, A. Pukhov, G. M. Pruna, and M. Purmohammadi, Phenomenology of charged scalars in the $\mathrm{CP}$ violating inert-doublet model, J. High Energy Phys. 04 (2013) 040.

[43] T. Abe and R. Sato, Quantum corrections to the spinindependent cross section of the inert doublet dark matter, J. High Energy Phys. 03 (2015) 109.

[44] N. Blinov, J. Kozaczuk, D. E. Morrissey, and A. de la Puente, Compressing the inert doublet model, Phys. Rev. D 93, 035020 (2016).

[45] M. A. Díaz, B. Koch, and S. Urrutia-Quiroga, Constraints to dark matter from inert Higgs doublet model, Adv. High Energy Phys. 2016, 8278375 (2016).

[46] G. Belanger, B. Dumont, A. Goudelis, B. Herrmann, S. Kraml, and D. Sengupta, Dilepton constraints in the inert doublet model from Run 1 of the LHC, Phys. Rev. D 91, 115011 (2015).

[47] A. Carmona and M. Chala, Composite dark sectors, J. High Energy Phys. 06 (2015) 105.

[48] S. Kanemura, M. Kikuchi, and K. Sakurai, Testing the dark matter scenario in the inert doublet model by future precision measurements of the Higgs boson couplings, Phys. Rev. D 94, 115011 (2016).
[49] B. Eiteneuer, A. Goudelis, and J. Heisig, The inert doublet model in the light of Fermi-LAT gamma-ray data: A global fit analysis, Eur. Phys. J. C 77, 624 (2017).

[50] A. Ilnicka, T. Robens, and T. Stefaniak, Constraining extended scalar sectors at the LHC and beyond, Mod. Phys. Lett. A 33, 1830007 (2018).

[51] J. Kalinowski, W. Kotlarski, T. Robens, D. Sokolowska, and A.F. Zarnecki, Benchmarking the inert doublet model for $e^{+} e^{-}$colliders, J. High Energy Phys. 12 (2018) 081.

[52] G. Belanger, F. Boudjema, A. Pukhov, and A. Semenov, micrOMEGAs_3: A program for calculating dark matter observables, Comput. Phys. Commun. 185, 960 (2014).

[53] G. Bélanger, F. Boudjema, A. Goudelis, A. Pukhov, and B. Zaldivar, micrOMEGAs5.0: Freeze-in, Comput. Phys. Commun. 231, 173 (2018).

[54] G. C. Branco, P. M. Ferreira, L. Lavoura, M. N. Rebelo, M. Sher, and J. P. Silva, Theory and phenomenology of two-Higgs-doublet models, Phys. Rep. 516, 1 (2012).

[55] M. Cirelli, N. Fornengo, and A. Strumia, Minimal dark matter, Nucl. Phys. B753, 178 (2006).

[56] L. L. Honorez and C. E. Yaguna, The inert doublet model of dark matter revisited, J. High Energy Phys. 09 (2010) 046.

[57] E. Aprile et al., Dark Matter Search Results from a One Ton-Year Exposure of XENON1T, Phys. Rev. Lett. 121, 111302 (2018).

[58] N. Baro, F. Boudjema, and A. Semenov, Full one-loop corrections to the relic density in the MSSM: A few examples, Phys. Lett. B 660, 550 (2008).

[59] N. Baro, F. Boudjema, and A. Semenov, Automatised full one-loop renormalisation of the MSSM. I. The Higgs sector, the issue of $\tan$ (beta) and gauge invariance, Phys. Rev. D 78, 115003 (2008).

[60] N. Baro, F. Boudjema, G. Chalons, and S. Hao, Relic density at one-loop with gauge boson pair production, Phys. Rev. D 81, 015005 (2010).

[61] G. Chalons and F. Domingo, Analysis of the Higgs potentials for two doublets and a singlet, Phys. Rev. D 86, 115024 (2012).

[62] A. Semenov, LanHEP: A package for the automatic generation of Feynman rules in field theory. Version 3.0, Comput. Phys. Commun. 180, 431 (2009).

[63] A. Semenov, LanHEP-A package for automatic generation of Feynman rules from the Lagrangian. Version 3.2, Comput. Phys. Commun. 201, 167 (2016).

[64] T. Hahn and M. Perez-Victoria, Automatized one loop calculations in four-dimensions and $D$-dimensions, Comput. Phys. Commun. 118, 153 (1999).

[65] T. Hahn, Generating Feynman diagrams and amplitudes with FeynArts 3, Comput. Phys. Commun. 140, 418 (2001).

[66] T. Hahn, Automatic loop calculations with FeynArts, FormCalc, and LoopTools, Nucl. Phys. B, Proc. Suppl. 89, 231 (2000).

[67] F. Boudjema, G. D. La Rochelle, and S. Kulkarni, Oneloop corrections, uncertainties and approximations in neutralino annihilations: Examples, Phys. Rev. D 84, 116001 (2011). 
[68] F. Boudjema, G. D. La Rochelle, and A. Mariano, Relic density calculations beyond tree-level, exact calculations versus effective couplings: The $Z Z$ final state, Phys. Rev. D 89, 115020 (2014).

[69] T. Han, H. Liu, S. Mukhopadhyay, and X. Wang, Dark matter blind spots at one-loop, J. High Energy Phys. 03 (2019) 080.
[70] S. Banerjee and N. Chakrabarty, A revisit to scalar dark matter with radiative corrections, J. High Energy Phys. 05 (2019) 150.

[71] D. Chowdhury and O. Eberhardt, Global fits of the two-loop renormalized two-Higgs-doublet model with soft $Z_{2}$ breaking, J. High Energy Phys. 11 (2015) 052. 